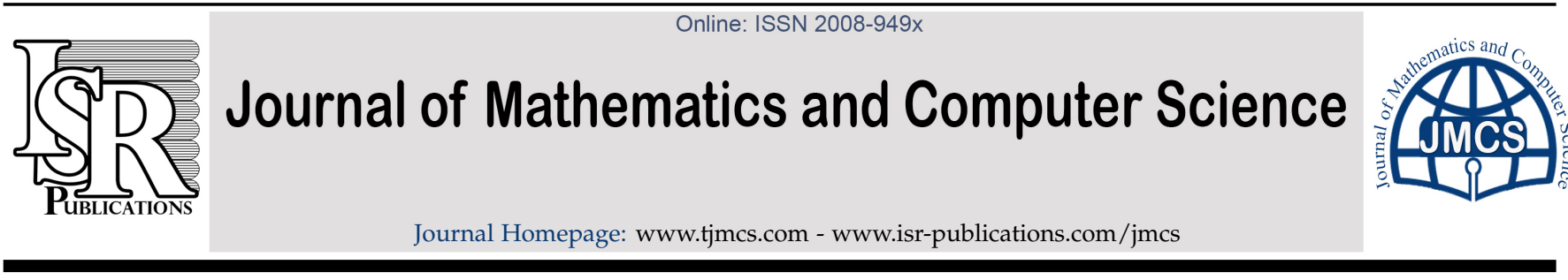

\title{
Generalization of Khan fixed point theorem
}

\author{
Hossein Piria , Samira Rahrovi ${ }^{\mathrm{a}}$, Poom Kumam ${ }^{\mathrm{b}, \mathrm{c}, \mathrm{d}, *}$ \\ ${ }^{a}$ Department of Mathematics, Basic Science Faculty, University of Bonab, Bonab, 5551761167, Iran. \\ ${ }^{b}$ KMUTT Fixed Point Research Laboratory, Department of Mathematics, Room SCL 802 Fixed Point Laboratory, Science Laboratory \\ Building, Faculty of Science, King Mongkuts University of Technology Thonburi (KMUTT), 126 Pracha Uthit Road, Bang Mod, Thung \\ Khru, Bangkok, 10140, Thailand. \\ ${ }^{c}$ KMUTT-Fixed Point Theory and Applications Research Group (KMUTT-FPTA), Theoretical and Computational Science Center (TaCS), \\ Science Laboratory Building, Faculty of Science, King Mongkuts University of Technology Thonburi (KMUTT), 126 Pracha Uthit Road, \\ Bang Mod, Thung Khru, Bangkok, 10140, Thailand. \\ ${ }^{d}$ Department of Medical Research, China Medical University Hospital, China Medical University, Taichung 40402, Taiwan.
}

\begin{abstract}
In this paper, we study some results of existence and uniqueness of fixed points for a class of mappings satisfying an inequality of rational expressions. Our main result extends and unifies the well-known results of Khan [M. S. Khan, Rend. Inst. Math. Univ. Trieste, 8 (1976), 69-72]. (C)2017 all rights reserved.
\end{abstract}

Keywords: Fixed point, metric space, Khan theorem.

2010 MSC: 74H10, 54H25.

\section{Introduction and preliminaries}

In the mid-sixties, fixed points results dealing with general contractive conditions with rational expressions were appeared. On of the well-known works in this direction were established by Khan [2] as follows:

Theorem 1.1. Let $(\mathrm{X}, \mathrm{d})$ be a complete metric space and let $\mathrm{T}: \mathrm{X} \rightarrow \mathrm{X}$ satisfies

$$
d(T x, T y) \leqslant k \frac{d(x, T x) d(x, T y)+d(y, T y) d(y, T x)}{d(x, T y)+d(T x, y)},
$$

where $\mathrm{k} \in[0,1)$ and $\mathrm{x}, \mathrm{y} \in \mathrm{X}$. Then $\mathrm{T}$ has a unique fixed point $\mathrm{x}^{*} \in \mathrm{X}$. Moreover, for all $\mathrm{x} \in \mathrm{X}$, the sequence $\left\{\mathrm{T}^{\mathrm{n}} \mathrm{x}\right\}$ converges to $x^{*}$.

It was shown by Fisher [1] that in (1.1) if the denominator vanishes, then $y=T y$ and $y=T x$ and consequently also the numerator vanishes. Moreover, we have $d(T x, T y)=d(x, y)$, and so the contractive

\footnotetext{
*Corresponding author

Email addresses: h.piri@bonabu.ac.ir (Hossein Piri), s.rahrovi@bonabu.ac.ir (Samira Rahrovi), poom.kumam@mail.kmutt.ac.th (Poom Kumam)

doi:10.22436/jmcs.017.01.07
} 
condition is not well-defined. In fact, it needed some extra conditions, that is,

$$
d(x, T y)+d(T x, y)=0 \text { implies that } d(T x, T y)=0 .
$$

Thus, the correct version of Theorem 1.1 can be stated as follows:

Theorem $1.2([1])$. Let $(\mathrm{X}, \mathrm{d})$ be a complete metric space and let $\mathrm{T}: \mathrm{X} \rightarrow \mathrm{X}$ satisfies

$$
d(T x, T y) \leqslant \begin{cases}k \frac{d(x, T x) d(x, T y)+d(y, T y) d(y, T x)}{d(x, T y)+d(T x, y)}, & \text { if } d(x, T y)+d(T x, y) \neq 0, \\ 0, & \text { if } d(x, T y)+d(T x, y)=0,\end{cases}
$$

where $\mathrm{k} \in[0,1)$ and $\mathrm{x}, \mathrm{y} \in \mathrm{X}$. Then $\mathrm{T}$ has a unique fixed point $\mathrm{x}^{*} \in \mathrm{X}$. Moreover, for all $\mathrm{x} \in \mathrm{X}$, the sequence $\left\{\mathrm{T}^{\mathrm{n}} \mathrm{x}\right\}$ converges to $x^{*}$.

Also by providing some examples, Fisher [1] showed the insufficiency of Khan's theorem. Recently, Redjel et al., by introducing $(\alpha, \beta)$-Meir-Keeler-Khan mapping have generalized the result of Fisher (see [3]).

In this paper, we introduce a new type of contraction satisfying an inequality of rational expressions and prove a new fixed point theorem concerning this type of contraction. Our result is real generalization of Khan fixed point theorem. The article includes an example showing that the obtained extension is significant.

\section{Main results}

Our main theorem is essentially inspired by Khan [2], Fisher [1], and Rhoades [4]. More precisely, we state and prove the following result.

Theorem 2.1. Let $(\mathrm{X}, \mathrm{d})$ be a complete metric space and let $\mathrm{T}: \mathrm{X} \rightarrow \mathrm{X}$ be a self-mapping such that for all $\mathrm{x}, \mathrm{y} \in \mathrm{X}$

$$
d(T x, T y) \leqslant \begin{cases}M(x, y)-\varphi(M(x, y)), & \text { if } \max \{d(x, T y), d(T x, y)\} \neq 0, \\ 0, & \text { if } \max \{d(x, T y), d(T x, y)\}=0,\end{cases}
$$

where

(a) $\varphi:[0, \infty) \rightarrow[0, \infty)$ is a lower semi-continuous function with $\varphi(t)=0$ if and only if $t=0$;

(b) $M(x, y)=\frac{d(x, T x) d(x, T y)+d(y, T y) d(y, T x)}{\max \{d(x, T y), d(T x, y)\}}$.

Then $T$ has a unique fixed point $x^{*} \in X$. Moreover, for all $x \in X$, the sequence $\left\{\mathrm{T}^{\mathrm{n}}(\mathrm{x})\right\}$ converges to $\mathrm{x}^{*}$.

Proof. Let $x_{0}=x \in X$. Put $x_{n+1}=T x_{n}=T^{n+1} x_{0}$ for all $n=0,1,2, \cdots$. If there exists $n \in \mathbb{N}$ such that $x_{n}=x_{n-1}$, then $x_{n-1}$ is a fixed point of T. This completes the proof. Therefore, we suppose $x_{n} \neq x_{n-1}$ for all $n \in \mathbb{N}$. We shall divide the proof into two cases.

Cases 1. Assume that $\max \left\{d\left(x_{n-1}, T x_{n}\right), d\left(T x_{n-1}, x_{n}\right)\right\} \neq 0$ for all $n \in \mathbb{N}$. Then, from (2.1) we have

$$
\begin{aligned}
d\left(x_{n}, x_{n+1}\right)= & d\left(T x_{n-1}, T x_{n}\right) \\
\leqslant & \frac{d\left(x_{n-1}, T x_{n-1}\right) d\left(x_{n-1}, T x_{n}\right)+d\left(x_{n}, T x_{n}\right) d\left(x_{n}, T x_{n-1}\right)}{\max \left\{d\left(x_{n-1}, T x_{n}\right), d\left(T x_{n-1}, x_{n}\right)\right\}} \\
& -\varphi\left(\frac{d\left(x_{n-1}, T x_{n-1}\right) d\left(x_{n-1}, T x_{n}\right)+d\left(x_{n}, T x_{n}\right) d\left(x_{n}, T x_{n-1}\right)}{\max \left\{d\left(x_{n-1}, T x_{n}\right), d\left(T x_{n-1}, x_{n}\right)\right\}}\right) \\
= & \frac{d\left(x_{n-1}, x_{n}\right) d\left(x_{n-1}, x_{n+1}\right)+d\left(x_{n}, x_{n+1}\right) d\left(x_{n}, x_{n}\right)}{\max \left\{d\left(x_{n-1}, x_{n+1}\right), d\left(x_{n}, x_{n}\right)\right\}} \\
& -\varphi\left(\frac{d\left(x_{n-1}, x_{n}\right) d\left(x_{n-1}, x_{n+1}\right)+d\left(x_{n}, x_{n+1}\right) d\left(x_{n}, x_{n}\right)}{\max \left\{d\left(x_{n-1}, x_{n+1}\right), d\left(x_{n}, x_{n}\right)\right\}}\right) \\
= & d\left(x_{n-1}, x_{n}\right)-\varphi\left(d\left(x_{n-1}, x_{n}\right)\right) .
\end{aligned}
$$


Since $\varphi\left(d\left(x_{n-1}, x_{n}\right)\right)>0$, hence $\left\{d\left(x_{n}, x_{n+1}\right)\right\}$ is monotonic nonincreasing sequence which is bounded below. So, there exists $\gamma \geqslant 0$ such that

$$
\lim _{n \rightarrow \infty} d\left(x_{n}, x_{n+1}\right)=\gamma=\lim _{n \rightarrow \infty} d\left(x_{n-1}, x_{n}\right) .
$$

We claim that $\gamma=0$. Arguing by contradiction, we assume that $\gamma>0$. So from (a), we have

$$
0<\varphi(\gamma) \leqslant \liminf _{n \rightarrow \infty} \varphi\left(d\left(x_{n}, x_{n+1}\right)\right) .
$$

Taking limit superior as $n \rightarrow \infty$ to each side of the (2.2), we get

$$
\begin{aligned}
\limsup _{n \rightarrow \infty} \varphi\left(d\left(x_{n}, x_{n+1}\right)\right) & \leqslant \limsup _{n \rightarrow \infty} d\left(x_{n-1}, x_{n}\right)+\limsup _{n \rightarrow \infty}\left[-\varphi\left(d\left(x_{n-1}, x_{n}\right)\right)\right] \\
& =\limsup _{n \rightarrow \infty} d\left(x_{n-1}, x_{n}\right)-\liminf _{n \rightarrow \infty} \varphi\left(d\left(x_{n-1}, x_{n}\right)\right) .
\end{aligned}
$$

So, from (2.3), (2.4), and (2.5), we obtain $0<\gamma \leqslant \gamma-\varphi(\varphi)<\gamma$. It is a contradiction. Therefore, we have

$$
\lim _{n \rightarrow \infty} d\left(x_{n}, T x_{n}\right)=\lim _{n \rightarrow \infty} d\left(x_{n}, x_{n+1}\right)=0 .
$$

Now, we claim that, $\left\{x_{n}\right\}_{n=1}^{\infty}$ is a Cauchy sequence. Arguing by contradiction, we assume that there exist $\epsilon>0$, and the sequences $\{p(n)\}_{\mathfrak{n}=1}^{\infty}$ and $\{\mathbf{q}(\mathfrak{n})\}_{\mathfrak{n}=1}^{\infty}$ of natural numbers such that

$$
p(n)>q(n)>n, \quad d\left(x_{p(n)}, x_{q(n)}\right) \geqslant \epsilon, d\left(x_{p(n)-1}, x_{q(n)}\right)<\epsilon, \quad \forall n \in \mathbb{N} .
$$

From the triangle inequality, we get

$$
\begin{aligned}
\epsilon \leqslant \mathrm{d}\left(\mathrm{x}_{\mathfrak{p}(\mathfrak{n})}, x_{\mathbf{q}(\mathfrak{n})}\right) & \leqslant \mathrm{d}\left(\mathrm{x}_{\mathbf{p}(\mathfrak{n})}, x_{\mathfrak{p}(\mathfrak{n})-1}\right)+\mathrm{d}\left(\mathrm{x}_{\mathfrak{p}(\mathfrak{n})-1}, x_{\mathbf{q}(\mathfrak{n})}\right) \\
& <\mathrm{d}\left(T x_{\mathfrak{p}(\mathfrak{n})-1}, x_{\mathfrak{p}(\mathfrak{n})-1}\right)+\epsilon, \quad \forall \mathfrak{n} \in \mathbb{N} .
\end{aligned}
$$

Thus from (2.6), (2.7), and the sandwich theorem, we get

$$
\limsup _{n \rightarrow \infty} d\left(x_{p(n)}, x_{q(n)}\right)=\epsilon
$$

By the triangle inequality, for all $n \in \mathbb{N}$, we have the following two inequalities:

$$
\begin{aligned}
d\left(x_{p(n)}, x_{q(n)}\right) & \leqslant d\left(x_{p(n)}, x_{q(n)+1}\right)+d\left(x_{q(n)+1}, x_{q(n)}\right) \\
& \leqslant d\left(x_{p(n)}, x_{q(n)}\right)+d\left(x_{q(n)+1}, x_{q(n)}\right)+d\left(x_{q(n)+1}, x_{q(n)}\right) \\
& =d\left(x_{p(n)}, x_{q(n)}\right)+2 d\left(x_{q(n)+1}, x_{q(n)}\right) .
\end{aligned}
$$

Letting $n \rightarrow \infty$ in inequalities (2.9) and using (2.6) and (2.8), we obtain

$$
\lim _{n \rightarrow \infty} d\left(x_{p(n)}, x_{q(n)+1}\right)=\epsilon .
$$

So, there exists $\mathrm{N}_{1} \in \mathbb{N}$ such that

$$
\mathrm{d}\left(\mathrm{x}_{\mathrm{p}(\mathrm{n})}, T \mathrm{x}_{\mathbf{q}(\mathrm{n})}\right)=\mathrm{d}\left(\mathrm{x}_{\mathrm{p}(\mathrm{n})}, \mathrm{x}_{\mathbf{q}(\mathrm{n})+1}\right)>\frac{\epsilon}{2}, \quad \forall \mathrm{n} \geqslant \mathrm{N}_{1} .
$$

Hence

$$
\max \left\{\mathrm{d}\left(\mathrm{x}_{\mathbf{p}(\mathrm{n})}, \mathrm{T} \mathrm{x}_{\mathbf{q}(\mathrm{n})}\right), \mathrm{d}\left(\mathrm{T} \mathrm{x}_{\mathrm{p}(\mathrm{n})}, \mathrm{x}_{\mathbf{q}(\mathrm{n})}\right)\right\}>\frac{\epsilon}{2}, \quad \forall \mathrm{n} \geqslant \mathrm{N}_{1} .
$$

Again by the triangle inequality, for all $n \in \mathbb{N}$, we have the following two inequalities:

$$
d\left(x_{p(n)}, x_{q(n)}\right) \leqslant d\left(x_{p(n)}, x_{p(n)+1}\right)+d\left(x_{p(n)+1}, x_{q(n)+1}\right)+d\left(x_{q(n)+1}, x_{q(n)}\right)
$$


and

$$
d\left(x_{p(n)+1}, x_{q(n)+1}\right) \leqslant d\left(x_{p(n)+1}, x_{p(n)}\right)+d\left(x_{p(n)}, x_{q(n)}\right)+d\left(x_{q(n)}, x_{q(n)+1}\right) .
$$

Letting $n \rightarrow \infty$ in inequalities (2.12) and (2.13), using (2.6) and (2.10), we obtain

$$
\limsup _{n \rightarrow \infty} d\left(x_{p(n)+1}, x_{q(n)+1}\right)=\epsilon .
$$

So, there exists $N_{2} \in \mathbb{N}$ such that

$$
\mathrm{d}\left(\mathrm{x}_{\mathrm{p}(\mathrm{n})+1}, \mathrm{x}_{\mathbf{q}(\mathrm{n})+1}\right)>\frac{\epsilon}{2}, \quad \forall \mathrm{n} \geqslant \mathrm{N}_{2} .
$$

So for all $n \geqslant N=\max \left\{N_{1}, N_{2}\right\}$ from (2.11) and (2.14), we have

$$
\begin{aligned}
& \frac{\epsilon}{2}<d\left(x_{p(n)+1}, x_{q(n)+1}\right) \\
& =\mathrm{d}\left(\mathrm{T} x_{\mathrm{p}(\mathrm{n})}, \mathrm{T} \mathrm{x}_{\mathrm{q}(\mathrm{n})}\right) \\
& \leqslant \frac{d\left(x_{p(n)}, T x_{p(n)}\right) d\left(x_{p(n)}, T x_{q(n)}\right)+d\left(x_{q(n)}, T x_{q(n)}\right) d\left(x_{q(n)}, T x_{p(n)}\right)}{\max \left\{d\left(x_{p(n)}, T x_{q(n)}\right), d\left(T x_{p(n)}, x_{q(n)}\right)\right\}} \\
& -\varphi\left(\frac{d\left(x_{p(n)}, T x_{p(n)}\right) d\left(x_{p(n)}, T x_{q(n)}\right)+d\left(x_{q(n)}, T x_{q(n)}\right) d\left(x_{q(n)}, T x_{p(n)}\right)}{\max \left\{d\left(x_{p(n)}, T x_{q(n)}\right), d\left(T x_{p(n)}, x_{q(n)}\right)\right\}}\right) \\
& \leqslant \frac{d\left(x_{p(n)}, T x_{p(n)}\right) d\left(x_{p(n)}, T x_{q(n)}\right)+d\left(x_{q(n)}, T x_{q(n)}\right) d\left(x_{q(n)}, T x_{p(n)}\right)}{\max \left\{d\left(x_{p(n)}, T x_{q(n)}\right), d\left(T x_{p(n)}, x_{q(n)}\right)\right\}} .
\end{aligned}
$$

Since for all $n \geqslant N, \max \left\{d\left(x_{p(n)}, T x_{q(n)}\right), d\left(T x_{p(n)}, x_{q(n)}\right)\right\} \geqslant \frac{\epsilon}{2}$, we have

$$
\begin{aligned}
0 & \leqslant \frac{d\left(x_{p(n)}, T x_{p(n)}\right) d\left(x_{p(n)}, T x_{q(n)}\right)+d\left(x_{q(n)}, T x_{q(n)}\right) d\left(x_{q(n)}, T x_{p(n)}\right)}{\max \left\{d\left(x_{p(n)}, T x_{q(n)}\right), d\left(T x_{p(n)}, x_{q(n)}\right)\right\}} \\
& \leqslant 2 \frac{d\left(x_{p(n)}, T x_{p(n)}\right) d\left(x_{p(n)}, T x_{q(n)}\right)+d\left(x_{q(n)}, T x_{q(n)}\right) d\left(x_{q(n)}, T x_{p(n)}\right)}{\epsilon} .
\end{aligned}
$$

So from (2.6), we get

$$
\lim _{n \rightarrow \infty} \frac{d\left(x_{p(n)}, T x_{p(n)}\right) d\left(x_{p(n)}, T x_{q(n)}\right)+d\left(x_{q(n)}, T x_{q(n)}\right) d\left(x_{q(n)}, T x_{p(n)}\right)}{\max \left\{d\left(x_{p(n)}, T x_{q(n)}\right), d\left(T x_{p(n)}, x_{q(n)}\right)\right\}}=0 .
$$

It follows from (2.15) and (2.16) that $\epsilon \leqslant 0$. This contradiction shows that $\left\{x_{n}\right\}$ is a cauchy sequence. By completeness of $(X, d),\left\{x_{n}\right\}$ converges to some point $x^{*}$ in $X$. Therefore

$$
\lim _{n \rightarrow \infty} d\left(x_{n}, x^{*}\right)=0
$$

We only have the following two cases

(I) $\forall \mathrm{n} \in \mathbb{N} \quad \exists i_{n} \in \mathbb{N}, \quad i_{n}>i_{n-1}, \quad i_{0}=1$ and $x_{i_{n+1}}=T x^{*}$;

(II) $\exists \mathrm{N} \in \mathbb{N}, \quad \forall \mathrm{n} \geqslant \mathrm{N}, \mathrm{d}\left(x_{\mathrm{n}}, \mathrm{T} x^{*}\right)>0$.

In the first case from (2.17), we have

$$
x^{*}=\lim _{n \rightarrow \infty} x_{i_{n+1}}=\lim _{n \rightarrow \infty} \mathrm{T} x^{*}=\mathrm{T} x^{*} .
$$

In the second case, for all $n \geqslant N$, we have

$$
\max \left\{d\left(x_{n}, T x^{*}\right), d\left(T x_{n}, x^{*}\right)\right\}>0 .
$$


So from (2.1), we get

$$
\begin{aligned}
d\left(x_{n+1}, T x^{*}\right)= & d\left(T x_{n}, T x^{*}\right) \\
\leqslant & \frac{d\left(x_{n}, T x_{n}\right) d\left(x_{n}, T x^{*}\right)+d\left(x^{*}, T x^{*}\right) d\left(x^{*}, T x_{n}\right)}{\max \left\{d\left(x_{n}, T x^{*}\right), d\left(T x_{n}, x^{*}\right)\right\}} \\
& -\varphi\left(\frac{d\left(x_{n}, T x_{n}\right) d\left(x_{n}, T x^{*}\right)+d\left(x^{*}, T x^{*}\right) d\left(x^{*}, T x_{n}\right)}{\max \left\{d\left(x_{n}, T x^{*}\right), d\left(T x_{n}, x^{*}\right)\right\}}\right) \\
\leqslant & \frac{d\left(x_{n}, T x_{n}\right) d\left(x_{n}, T x^{*}\right)+d\left(x^{*}, T x^{*}\right) d\left(x^{*}, T x_{n}\right)}{\max \left\{d\left(x_{n}, T x^{*}\right), d\left(T x_{n}, x^{*}\right)\right\}} .
\end{aligned}
$$

Since $\lim _{n \rightarrow \infty} d\left(x_{n}, T x^{*}\right)=d\left(x^{*}, T x^{*}\right)$, so from (2.6), (2.17), and taking limits as $n \rightarrow \infty$ to each side of the (2.18), we get $d\left(x^{*}, T x^{*}\right) \leqslant 0$ and hence $d\left(x^{*}, T x^{*}\right)=0$. Now, we show that $T$ has a unique fixed point. For this, we assume that $y^{*}$ is another fixed point of $T$ in $X$ such that $d\left(x^{*}, y^{*}\right)>0$. Therefore

$$
\max \left\{d\left(x^{*}, T y^{*}\right), d\left(T x^{*}, y^{*}\right)\right\}=d\left(x^{*}, y^{*}\right)>0 .
$$

So from (2.1), we get

$$
\begin{aligned}
d\left(x^{*}, y^{*}\right) & =d\left(T x^{*}, T y^{*}\right) \\
& \leqslant \frac{d\left(x^{*}, T x^{*}\right) d\left(x^{*}, T y^{*}\right)+d\left(y^{*}, T y^{*}\right) d\left(y^{*}, T x^{*}\right)}{\max \left\{d\left(x^{*}, T y^{*}\right), d\left(T x^{*}, y^{*}\right)\right\}}-\varphi\left(\frac{d\left(x^{*}, T x^{*}\right) d\left(x^{*}, T y^{*}\right)+d\left(y^{*}, T y^{*}\right) d\left(y^{*}, T x^{*}\right)}{\max \left\{d\left(x^{*}, T y^{*}\right), d\left(T x^{*}, y^{*}\right)\right\}}\right) \\
& =\frac{0 \times d\left(x^{*}, T y^{*}\right)+0 \times d\left(y^{*}, T x^{*}\right)}{\max \left\{d\left(x^{*}, T y^{*}\right), d\left(T x^{*}, y^{*}\right)\right\}}-\varphi\left(\frac{0 \times d\left(x^{*}, T y^{*}\right)+0 \times d\left(y^{*}, T x^{*}\right)}{\max \left\{d\left(x^{*}, T y^{*}\right), d\left(T x^{*}, y^{*}\right)\right\}}\right) \\
& =0-\varphi(0)=0 .
\end{aligned}
$$

So from $(a)$, we get $d\left(x^{*}, y^{*}\right)=0$. This leads to a contradiction and hence $x^{*}=y^{*}$. This completes the proof.

Case 2. Assume that there exists $m \in \mathbb{N}$ such that

$$
\max \left\{\mathrm{d}\left(\mathrm{x}_{\mathrm{m}-1}, \mathrm{~T} x_{\mathrm{m}}\right), \mathrm{d}\left(\mathrm{T} x_{\mathrm{m}-1}, \mathrm{x}_{\mathrm{m}}\right)\right\}=0 .
$$

By condition (2.1), it follows that $d\left(T x_{m-1}, T x_{m}\right)=0$ and hence $x_{m}=T x_{m}$. This completes the proof of the existence of a fixed point of $\mathrm{T}$. The uniqueness follows as in Case 1.

Corollary 2.2. Let $(\mathrm{X}, \mathrm{d})$ be a complete metric space and let $\mathrm{T}: \mathrm{X} \rightarrow \mathrm{X}$ satisfy

$$
d(T x, T y) \leqslant \begin{cases}k \frac{d(x, T x) d(x, T y)+d(y, T y) d(y, T x)}{\max \{d(x, T y), d(T x, y)\}}, & \text { if } \max \{d(x, T y), d(T x, y)\} \neq 0, \\ 0, & \text { if } \max \{d(x, T y), d(T x, y)\}=0,\end{cases}
$$

for some $\mathrm{k} \in[0,1)$ and $\mathrm{x}, \mathrm{y} \in \mathrm{X}$. Then $T$ has a unique fixed point $\mathrm{x}^{*} \in \mathrm{X}$. Moreover, for all $\mathrm{x} \in \mathrm{X}$, the sequence $\left\{\mathrm{T}^{\mathrm{n}}(\mathrm{x})\right\}$ converges to $\mathrm{x}^{*} \in \mathrm{X}$.

Proof. It is sufficient to take $\varphi(t)=\tau t$ for all $t \in[0, \infty)$ and $\tau \in(0,1)$ and $k=1-\tau$ in Theorem 2.1.

Theorem 2.3. Let $(\mathrm{X}, \mathrm{d})$ be a complete metric space. Let $\mathrm{S}$ and $\mathrm{T}$ be mappings from $\mathrm{X}$ into itself such that $\mathrm{S}$ and $\mathrm{T}$ commute and $\mathrm{S}$ satisfies

$$
d(S x, S y) \leqslant \begin{cases}k \frac{d(x, S x) d(x, S y)+d(y, S y) d(y, S x)}{d(x, S y)+d(S x, y)}, & \text { if } d(x, S y)+d(S x, y) \neq 0, \\ 0, & \text { if } d(x, S y)+d(S x, y)=0\end{cases}
$$

where $\mathrm{k} \in[0,1)$ and $\mathrm{x}, \mathrm{y} \in \mathrm{X}$. Then $\mathrm{T}$ has a unique fixed point $\mathrm{x}^{*} \in \mathrm{X}$. 
Proof. By Theorem 2.2, $\mathrm{S}$ has a unique fixed point $x^{*} \in \mathrm{X}$. Then, we have

$$
\mathrm{S}\left(\mathrm{T} x^{*}\right)=\mathrm{T}\left(\mathrm{S} x^{*}\right)=\mathrm{T} x^{*},
$$

and so $T x^{*}$ is a fixed point of $S$. Therefore, by uniqueness of the fixed point of $S$ it must be that $T x^{*}=$ $x^{*}$.

Theorem $2.4([1])$. Let $(X, \mathrm{~d})$ be a complete metric space and let $\mathrm{T}: \mathrm{X} \rightarrow \mathrm{X}$ satisfies

$$
d(T x, T y) \leqslant \begin{cases}k \frac{d(x, T x) d(x, T y)+d(y, T y) d(y, T x)}{d(x, T y)+d(T x, y)}, & \text { if } d(x, T y)+d(T x, y) \neq 0, \\ 0, & \text { if } d(x, T y)+d(T x, y)=0,\end{cases}
$$

where $\mathrm{k} \in[0,1)$ and $\mathrm{x}, \mathrm{y} \in \mathrm{X}$. Then $\mathrm{T}$ has a unique fixed point $\mathrm{x}^{*} \in \mathrm{X}$. Moreover, for all $\mathrm{x} \in \mathrm{X}$, the sequence $\left\{T^{n}(x)\right\}$ converges to $x^{*}$.

Proof. Since $d(x, T y)+d(T x, y)>0$ implies that $\max \{d(x, T y), d(T x, y)\}>0$ and $d(x, T y)+d(T x, y)=0$ implies that $\max \{\mathrm{d}(\mathrm{x}, \mathrm{Ty}), \mathrm{d}(\mathrm{T} x, y)\}=0$, moreover

$$
\frac{d(x, T x) d(x, T y)+d(y, T y) d(y, T x)}{d(x, T y)+d(T x, y)} \leqslant \frac{d(x, T x) d(x, T y)+d(y, T y) d(y, T x)}{\max \{d(x, T y), d(T x, y)\}},
$$

so from Theorem 2.2 the proof is complete.

Example 2.5. Let $X=\left\{\frac{1}{n} \mid n \in \mathbb{N}\right\} \cup\{0\}$ and define a metric $d$ on $X$ by

$$
d(x, y)= \begin{cases}0, & \text { if } x=y, \\ 2, & \text { if }(x, y) \in\{(0,1),(1,0)\}, \\ \frac{3}{2}, & \text { if }(x, y) \in\left\{\left(1, \frac{1}{2}\right),\left(\frac{1}{2}, 1\right)\right\} \\ 1, & \text { if }(x, y) \in\left\{\left(0, \frac{1}{n+1}\right),\left(\frac{1}{n+1}, 0\right) \mid n \in \mathbb{N}\right\} \\ \max \{x, y\}, & \text { if }(x, y) \in\left\{\left(\frac{1}{m}, \frac{1}{n}\right) \mid m, n \in \mathbb{N}\right\}-\left\{\left(1, \frac{1}{2}\right),\left(\frac{1}{2}, 1\right)\right\}, x \neq y\end{cases}
$$

We rewrite $X$ as $X=\left\{\frac{1}{n+2} \mid n \in \mathbb{N}\right\} \cup\left\{0, \frac{1}{2}, 1\right\}$. Obviously for all $x, y \in X, d(x, y) \geqslant 0$ and $d(x, y)=d(y, x)$. To check the triangle inequality consider the following cases.

Case 1. Let $x=0, y=\frac{1}{2}, z=1$, then

$$
d(x, y)=\frac{3}{2}, d(x, z)=2, d(y, z)=\frac{3}{2} .
$$

Case 2. Let $x=0, y=\frac{1}{2}, z \in\left\{\frac{1}{n+2} \mid n \in \mathbb{N}\right\}$, then

$$
d(x, y)=1, d(x, z)=1, d(y, z)=\frac{1}{2} .
$$

Case 3. Let $x=0, y=1, z \in\left\{\frac{1}{n+2} \mid n \in \mathbb{N}\right\}$, then

$$
d(x, y)=2, d(x, z)=1, d(y, z)=1 .
$$

Case 4. Let $x=\frac{1}{2}, y=1, z \in\left\{\frac{1}{n+2} \mid n \in \mathbb{N}\right\}$, then

$$
d(x, y)=\frac{3}{2}, d(x, z)=\frac{1}{2}, d(y, z)=1 .
$$

Case 5. Let $x=0, y, z \in\left\{\frac{1}{n+2} \mid n \in \mathbb{N}\right\}$, then

$$
d(x, y)=1, d(x, z)=1, d(y, z) \leqslant \frac{1}{3} .
$$


Case 6. Let $x=\frac{1}{2}, y, z \in\left\{\frac{1}{n+2} \mid n \in \mathbb{N}\right\}$, then

$$
d(x, y)=\frac{1}{2}, d(x, z)=\frac{1}{2}, d(y, z) \leqslant \frac{1}{3} .
$$

Case 7. Let $x=1, y, z \in\left\{\frac{1}{n+2} \mid n \in \mathbb{N}\right\}$, then

$$
d(x, y)=1, d(x, z)=1, d(y, z) \leqslant \frac{1}{3} .
$$

Case 8. Let $x, y, z \in\left\{\frac{1}{n+2} \mid n \in \mathbb{N}\right\}$, then

$$
\max \{x, y\} \leqslant x+y \leqslant \max \{x, z\}+\max \{z, y\} .
$$

In all cases triangle inequality is clearly established. Then, $(X, d)$ is a metric space and obviously it is complete. Let $\mathrm{T}: \mathrm{X} \rightarrow \mathrm{X}$ be defined by

$$
\mathrm{Tx}= \begin{cases}0, & \text { if } x \neq 1 \\ \frac{1}{2}, & \text { if } x=1\end{cases}
$$

Now we consider the following cases:

Case I. Let $(x, y) \in\{(0,1),(1,0)\}$, then

$$
\begin{aligned}
& \mathrm{d}(\mathrm{T} 0, \mathrm{~T} 1)=\mathrm{d}\left(0, \frac{1}{2}\right)=1, \\
& \mathrm{~d}(0, \mathrm{~T} 1)+\mathrm{d}(1, \mathrm{~T} 0)=3 \text {, } \\
& d(0, \text { T0 }) d(0, T 1)+d(1, T 1) d(1, T 0)=3, \\
& \max \{d(0, T 1), d(1, T 0)\}=2 .
\end{aligned}
$$

Case II. Let $(x, y) \in\left\{\left(1, \frac{1}{2}\right),\left(\frac{1}{2}, 1\right) \mid n \in \mathbb{N}\right\}$, then

$$
\begin{aligned}
\mathrm{d}\left(\mathrm{T} 1, \mathrm{~T} \frac{1}{2}\right)=\mathrm{d}\left(\frac{1}{2}, 0\right) & =1, & \mathrm{~d}(1, \mathrm{~T} 1) \mathrm{d}\left(1, \mathrm{~T} \frac{1}{2}\right)+\mathrm{d}\left(\frac{1}{2}, \mathrm{~T} \frac{1}{2}\right) \mathrm{d}\left(\frac{1}{2}, \mathrm{~T} 1\right) & =3, \\
\mathrm{~d}\left(1, \mathrm{~T} \frac{1}{2}\right)+\mathrm{d}\left(\mathrm{T} 1, \frac{1}{2}\right) & =2, & \max \left\{\mathrm{d}\left(1, \mathrm{~T} \frac{1}{2}\right), \mathrm{d}\left(\mathrm{T} 1, \frac{1}{2}\right)\right\} & =2 .
\end{aligned}
$$

Case III. Let $(x, y) \in\left\{\left(0, \frac{1}{n+1}\right),\left(\frac{1}{n+1}, 0\right) \mid n \in \mathbb{N}\right\}$, then

$$
\begin{array}{rlrl}
d\left(T 0, T \frac{1}{n+1}\right) & =0, & d(0, T 0) d\left(0, T \frac{1}{n+1}\right)+d\left(\frac{1}{n+1}, T \frac{1}{n+1}\right) d\left(\frac{1}{n+1}, T 0\right) & =1, \\
d\left(0, T \frac{1}{n+1}\right)+d\left(T 0, \frac{1}{n+1}\right) & =1, & \max \left\{d\left(0, T \frac{1}{n+1}\right), d\left(T 0, \frac{1}{n+1}\right)\right\}=1 .
\end{array}
$$

Case V. Let $(x, y) \in\left\{\left(\frac{1}{m}, \frac{1}{n}\right) \mid m, n \in \mathbb{N}\right\}-\left\{\left(1, \frac{1}{2}\right),\left(\frac{1}{2}, 1\right)\right\}$, and $x \neq y$, then

$$
\begin{array}{rlrl}
\mathrm{d}\left(\mathrm{T} \frac{1}{\mathrm{~m}}, \mathrm{~T} \frac{1}{\mathrm{n}}\right) & =0, & \mathrm{~d}\left(\frac{1}{\mathrm{~m}}, \mathrm{~T} \frac{1}{\mathrm{~m}}\right) \mathrm{d}\left(\frac{1}{\mathrm{~m}}, \mathrm{~T} \frac{1}{\mathrm{n}}\right)+\mathrm{d}\left(\frac{1}{\mathrm{n}}, \mathrm{T} \frac{1}{\mathrm{n}}\right) \mathrm{d}\left(\frac{1}{\mathrm{n}}, \mathrm{T} \frac{1}{\mathrm{~m}}\right)=2, \\
\mathrm{~d}\left(\frac{1}{\mathrm{~m}}, \mathrm{~T} \frac{1}{\mathrm{n}}\right)+\mathrm{d}\left(\mathrm{T} \frac{1}{\mathrm{~m}}, \frac{1}{\mathrm{n}}\right) & =2, & \max \left\{\mathrm{d}\left(\frac{1}{\mathrm{~m}}, \mathrm{~T} \frac{1}{\mathrm{n}}\right), \mathrm{d}\left(\mathrm{T} \frac{1}{\mathrm{~m}}, \frac{1}{\mathrm{n}}\right)\right\} & =1 .
\end{array}
$$

In Case I, we have

$$
d(T x, T y)=\frac{d(x, T x) d(x, T y)+d(y, T y) d(y, T x)}{d(x, T y)+d(y, T x)}=1 .
$$

This proves that $T$ does not satisfy in assumption of Theorem 1.2, because there is no nonnegative number $k<1$ satisfying the equation (1.2). However in all cases, we have

$$
d(T x, T y) \leqslant k \frac{d(x, T x) d(x, T y)+d(y, T y) d(y, T x)}{\max \{d(x, T y), d(y, T x)\}}
$$

for all $k \in\left[\frac{2}{3}, 1\right)$. So by Theorem $2.2,0$ is the unique fixed point of $\mathrm{T}$. 


\section{Acknowledgment}

This project was supported by the Theoretical and Computational Science (TaCS) Center under Computational and Applied Science for Smart Innovation Research Cluster (CLASSIC), Faculty of Science, KMUTT.

\section{References}

[1] B. Fisher, On a theorem of Khan, Riv. Math. Univ. Parma., 4 (1978), 135-137. 1, 1.2, 1, $2,2.4$

[2] M. S. Khan, A fixed point theorem for metric spaces, Rend. Inst. Math. Univ. Trieste, 8 (1976), 69-72.1, 2

[3] N. Redjel, A. Dehici, E. Karapinar, I. M. Erhan, Fixed point theorems for (alpha, psi)-Meir-Keeler-Khan mappings, J. Nonlinear Sci. Appl., 8 (2015), 955-964. 1

[4] B. E. Rhoades, Some theorems on weakly contractive maps, Nonlinear Anal., 47 (2001), 2683-2693.2 